\title{
APPLICATION OF TIME-DELAY RECONSTRUCTION METHOD FOR EXAMINATION OF SPATIAL INHOMOGENEITY OF DYNAMICAL SYSTEMS
}

\author{
A. ZUBRZYCKI \\ Department of Physics, Technical University \\ Malczewskiego 29, 26-600 Radom, Poland \\ AND A. SUKIENNICKI \\ Institute of Physics, Warsaw University of Technology \\ Koszykowa 75, 00-662 Warsaw, Poland \\ and \\ Institute of Physics, Polish Academy of Sciences \\ Al. Lotników 32/46, 02-668 Warsaw, Poland
}

(Received November 13, 1996; in final form February 18, 1997)

Three spatially extended one-dimensional dynamical systems are examined by numerical simulations and the time-delay technique is applied to find their dynamics at different positions in space. On the basis of this technique the correlation dimension is calculated from time series obtained at different positions. It is found that the value of the correlation dimension may vary from one position to another, reflecting the spatial inhomogeneity of the system.

PACS numbers: $05.45 .+b$

The time-delay reconstruction method, proposed some time ago by Takens [1] on the basis of an embedding theorem, allows a characterization of a dynamical system from a time series of a single component of the state. Reconstruction of the original attractor by this technique can be done for systems with a finite number of degrees of freedom, like iterated maps or systems described by ordinary differential equations. It has also been used successfully for some infinite-order systems [2]. Recently, it has been shown [3] that in the case of infinite-order spatially extended systems described by partial differential equations, reconstructions of the attractor from the time series obtained at different points of the space may give different results. It is especially expected, when the system is spatially inhomogeneous, because of, for example, boundaries or existence of spatially coherent structures. For 
such a system the time-delay reconstruction does not seem to be a useful method to find a global measure of the dynamics, e.g., a global (one for the whole system) value of the correlation dimension or of the largest Lyapunov exponent. However, the method may still be used to characterize local properties of the attractor. In the paper [3], the time-delay technique was applied to find the values of the local correlation dimension from the time series taken at different positions of the space. It was shown that these values may vary from one position to another. It suggests that the correlation dimension as a function of position may be a good measure of a spatial inhomogeneity of the system. Having all this in mind, we have examined, by numerical simulations, three spatially extended, one-dimensional systems, the first two of them, a Landau-Lifshitz equation and a driven damped nonlinear Schrödinger equation, containing the spatially coherent structure in the form of a single solitary wave (this solitary wave can propagate for the first system and it is static for the second one), and the third, a Ginzburg-Landau equation-system without the solitary wave. We have examined the spatial inhomogeneity of these systems by a Fourier transform in non-chaotic states and by the estimation of the local correlation dimension for the chaotic ones.

The first system under investigation is a model of the uniaxial ferromagnet treated as a one-dimensional continuous chain of magnetic vectors $M\left(|M|=M_{0}\right)$ extended along the $x$ axis of a Cartesian system. It is assumed that the uniaxial anisotropy axis is parallel to the $z$ direction and that the external oscillating field $H_{z}=H_{0} \cos \omega_{0} t$ is applied. The dynamics of the magnetization can be described by the following Landau-Lifshitz system of equations for the $\mu=M / M_{0}$ vector components [4]:

$$
\begin{aligned}
& \frac{1+\alpha^{2}}{\gamma} \mu_{x}=\alpha\left(\mu_{y}^{2}+\mu_{z}^{2}\right) H_{\mathrm{e} x}-\left(\alpha \mu_{x} \mu_{y}+\mu_{z}\right) H_{\mathrm{e} y}-\left(\alpha \mu_{x} \mu_{z}-\mu_{y}\right) H_{\mathrm{e} z}, \\
& \frac{1+\alpha^{2}}{\gamma} \mu_{y}=-\alpha\left(\mu_{x} \mu_{y}-\mu_{z}\right) H_{\mathrm{e} x}+\alpha\left(\mu_{x}^{2}+\mu_{z}^{2}\right) H_{\mathrm{e} y}-\left(\alpha \mu_{y} \mu_{z}+\mu_{x}\right) H_{\mathrm{e} z}, \\
& \frac{1+\alpha^{2}}{\gamma} \mu_{z}=-\left(\alpha \mu_{x} \mu_{z}+\mu_{y}\right) H_{\mathrm{e} x}-\left(\alpha \mu_{y} \mu_{z}-\mu_{x}\right) H_{\mathrm{e} y}+\alpha\left(\mu_{x}^{2}+\mu_{y}^{2}\right) H_{\mathrm{e} z},
\end{aligned}
$$

where the components of the effective field are

$$
\begin{aligned}
& H_{\mathrm{e} x}=\frac{2 K}{M_{0}} \frac{\partial^{2} \mu_{x}}{\partial x^{2}}-4 \pi M_{0} \mu_{x}, \quad H_{\mathrm{e} y}=\frac{2 K}{M_{0}} \frac{\partial^{2} \mu_{y}}{\partial x^{2}}, \\
& H_{\mathrm{e} z}=\frac{2 K}{M_{0}} \frac{\partial^{2} \mu_{z}}{\partial x^{2}}+\frac{2 K}{M_{0}} \mu_{z}+H_{0} \cos \omega_{0} t
\end{aligned}
$$

and $\gamma(>0)$ is the gyromagnetic ratio, $\alpha$ is the Gilbert damping constant, $K$ is the uniaxial anisotropy constant, $4 \pi M_{x}$ is the demagnetizing field. A dot over the symbols denotes differentiation over the time and $x$ is measured in units of $\sqrt{A / K}$, where $A$ is the exchange constant.

The second system is a driven damped nonlinear Schrödinger equation in the following form [5]:

$$
\psi_{t}=\mathrm{i} \frac{\partial^{2} \psi}{\partial x^{2}}+2 \mathrm{i}|\psi|^{2} \psi-b \psi-a \exp \left(\mathrm{i} \omega_{0} t\right) .
$$


The forcing field is characterized by the amplitude $a$ and frequency $\omega_{0}$ and the dissipation is measured by the $b$ parameter. All these parameters are real numbers.

The third system is a complex Ginzburg-Landau equation [6-9] in the following form:

$$
\psi_{t}=c_{0} \psi+\left(1+\mathrm{i} c_{1}\right) \frac{\partial^{2} \psi}{\partial x^{2}}-\left(1+\mathrm{i} c_{2}\right)|\psi|^{2} \psi
$$

where the parameters $c_{0}, c_{1}$ and $c_{2}$ are real numbers.

The system of equations (1) and the equations (3) and (4) were solved numerically by means of the 3rd order Adams-Bashforth-Moulton predictor-corrector method with a fixed time step $\Delta t=0.002 \mathrm{~ns}, \Delta t=0.01$, and $\Delta t=0.09$ (arbitrary units), respectively. For Eqs. (1) the pinning was assumed, while for Eqs. (3, 4), the two types of boundary conditions, pinned and periodic, were applied. The numerical integration was performed in a domain $-L \leq x \leq L$, with $L=10$ (in units of $\sqrt{A / K}$ ), $L=8.1 \pi, L=300$ (arbitrary units), for Eqs. (1), (3), (4), respectively and the second space derivatives were calculated by a pseudo-spectral method [10] for all variables except $\mu_{z}$ for which a 6 th order finite difference method was applied. A brief discussion of a validation of this method for each system is given in previous papers $[4,12,13]$. As an initial condition a single Bloch domain wall in the form $\mu_{x}(x, 0)=0, \mu_{y}(x, 0)=\operatorname{sech}(x), \mu_{z}=-\tanh (x)$ [11], was taken for Eqs. (1) and a single soliton, $\psi(\mathrm{x}, 0)=0.6 \operatorname{sech}(0.6 x) \exp (-\mathrm{i} \pi / 2)$ [5], for Eq. (3). For Eq. (4) a random initial space distribution of the amplitude $|\psi|$ was assumed. For the three systems the integration was performed long enough to reach an attractor and an initial transient was discarded before analyzing the data. The following parameters were fixed during the numerical simulations: $\omega_{0}=20 \mathrm{GHz}, \gamma=$ $1.76 \times 10^{7} 1 /(\mathrm{s} \mathrm{Oe}), 4 \pi \mathrm{M}_{0}=1700 \mathrm{Oe}, 2 \mathrm{~K} / \mathrm{M}_{0}=80 \mathrm{Oe}, \alpha=0.01$ for Eqs. (1), $\omega_{0}=1.0, b=0.1$ (all in arbitrary units) for Eq. (3), and $c_{0}=0.2, c_{1}=-1.0$ for Eq. (4). For the first two systems the amplitudes of the driving fields, $H_{0}$ for Eqs. (1) and $\boldsymbol{a}$ for Eq. (3), were varied. In the case of Eq. (4) the nonlinearity parameter $c_{2}$ was treated as the driving field and also was varied. The character of the solutions was examined on the basis of time series obtained at 129 grid points numbered from -64 to 64 , so that the point number 0 referred to the center of the particular system.

For all systems considered, roads to chaos were analyzed and then the chaotic states were examined with increasing amplitudes of the driving fields. Complete results are available in a series of previous papers $[4,12,13]$. Here we recapitulate some of them altogether, with emphasizing the dependence of the solutions on positions in space. Let us start with the first two systems. In the case of Eqs. (1) the system approaches the chaotic state after a sequence of periodic and quasi-periodic oscillations, while in the case of Eq. (3), we have a typical process of period-doubling bifurcations. In both cases, as long as the system is in periodic state, the character of the oscillations in the particular positions changes similarly with increasing distance from the system center (in the periodic regime the single solitary wave existing in both systems is static, so the center of the systems coincide with the center of the solitary wave). In the case of Eqs. (1) the oscillations in the center of the system are purely sinusoidal (Fig. 1a), while beyond the center, the successive higher harmonics appear and grow with increasing distance 
from the system center. Especially the amplitude of the second harmonic at $2 \omega_{0}$ increases gradually until it dominates over the first one at $\omega_{0}$. This process is seen in Fig. 1, which shows the spectrums of the oscillations of the $\mu_{x}$ variable at different space positions for $H_{0}=25 \mathrm{Oe}$. A similar process we have in the case of Eq. (3), except that the oscillations in the system center are not purely sinusoidal, so the spectrum of $|\psi|$ in this position consists of a sequence of evenly spaced harmonics (Fig. 2 [12]). Beyond the center, the amplitude of the first harmonic decreases, while the amplitudes of some higher harmonics, especially of the second one, increase with growing distance from the system center. The time wave forms of the amplitude $|\psi|$ of the solution of Eq. (3) for $\alpha=0.135$ at different positions are depicted in Fig. 2. It is common for both systems that the greater is the amplitude of the driving field, the amplitudes of the higher harmonics grow faster with increasing distance from the system center. Moreover, the transient time, after which the systems approach the periodic states, is longer for positions more distant from the center.
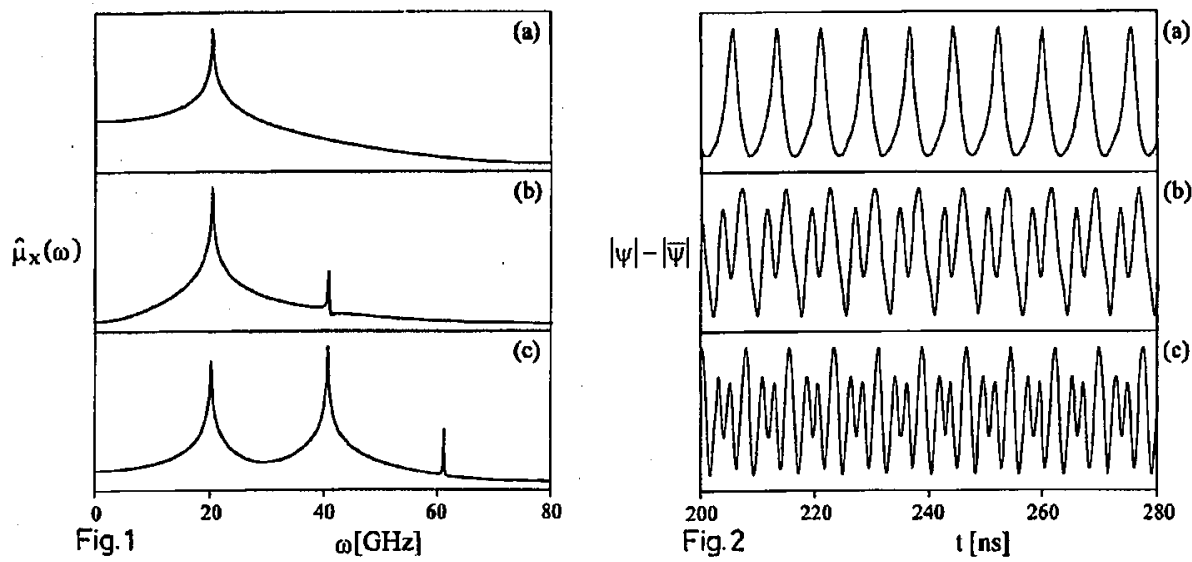

Fig. 1. Spectrum of the first component $\mu_{x}$ of the solution of Eqs. (1) for $H_{0}=25$ Oe at grid point number (a) 0 , (b) 30 , (c) 58 .

Fig. 2. Time wave form of the amplitude $|\psi|$ of the solution of Eq. (3) for $\alpha=0.135$ at grid point number (a) 0 , (b) 30 , (c) $50 .|\bar{\psi}|$ denotes an average amplitude in a time series.

When the amplitude of the driving field $H_{0}$ exceeds $H_{0} \approx 32 \mathrm{Oe}$, the oscillations at the center of the system described by Eqs. (1) are still purely sinusoidal, but beyond the center the motion is quasi-periodic, there appear new harmonics at the frequencies $0.5 \omega_{0} \pm \omega^{\prime}$ and $1.5 \omega_{0} \pm \omega^{\prime}$ (where $\omega^{\prime} \approx 0.42 \mathrm{GHz}$ is incommensurate with $\omega_{0}$ ) the amplitudes of which grow with the distance from the wall center. It means that the solution is 2-periodic with the base frequencies $0.5 \omega_{0}$ and $\omega^{\prime}$. This is illustrated by Fig. 3 for the first component $\mu_{x}$ of the solution at grid point number 58 and for $H_{0}=32.7 \mathrm{Oe}$.

The chaotic states appear for $H_{0} \approx 34.4 \mathrm{Oe}$ and $\alpha \approx 0.148$ in the case of Eqs. (1) and Eq. (3), respectively. In the chaotic regime a difference between 



Fig. 3. First component $\mu_{x}$ of the solution of Eqs. (1) at grid point number 58 for $H_{0}=32.7$ Oe. (a) Two-dimensional phase portrait $\mu_{x}\left(t_{i}\right)$ vs. $\mu_{x}\left(t_{i}+\tau\right)$ reconstructed from a time series. Time between successive data is $\Delta t=0.004 \mathrm{~ns}, t_{i}=i \Delta t$, and a delay time $\tau=0.22$ ns. (b) Spectrum of $\mu_{x}$.

the two systems appears important: the solitary wave existing in the system (1) (domain wall) moves along the $x$ axis, while the solitary wave of the system (3) is still static and only its amplitude oscillates chaotically.

For Eq. (4), as long as $1+c_{1} c_{2}>0$, a homogeneous solution $\psi=$ $\sqrt{c_{0}} \exp \left(-\mathrm{i} c_{0} c_{2} t\right)$ was found to be an attractor. When $1+c_{1} c_{2}$ becomes negative ( $c_{2}>1$ in our simulation), the homogeneous solution looses its linear stability and the system turns into a state of a "weak" chaos called a "phase turbulence". When $c_{2} \approx 1.4$, the system approaches a much "stronger" chaotic state called an "amplitude turbulence". The phase turbulent state is characterized by small fluctuations of the amplitude $|\psi|$ around $\sqrt{c_{0}}$. In the amplitude turbulent state the system exhibits large amplitude fluctuations and "defects" - regions, where the amplitude takes small values of nearly zero.

The spatial inhomogeneity of the all three systems was examined quantitatively by the estimation of the local correlation dimension $D_{\mathrm{C}}$ from time series taken at different grid points. To calculate the correlation dimension, a modified Grassberger-Procaccia algorithm [14] was used. It is based on the reconstruction of the attractor by the time-delay technique. In the first step the set of $N$ vectors in $m$-dimensional space

$$
\overline{\boldsymbol{X}}(i)=\left(x\left(t_{i}\right), x\left(t_{i}+\tau\right), \ldots, x\left(t_{i}+(m-1) \tau\right)\right)
$$

was constructed from the time series $x\left(t_{i}\right)$, where $t_{i}=i \Delta T(2 \leq i \leq N)$ and 
$\Delta T$ is the time between the successive data. Then $M<N$, reference vectors were randomly chosen and about each of these vectors, it was found the radius $\varepsilon(j)$ of the smallest $m$-dimensional sphere containing exactly $j$ vectors $(1 \leq j<$ $N-2 K$ ), where $K$ is the number of vectors skipped at each side of the reference vector to ensure that the vectors considered are sufficiently independent [15]). The radius $\varepsilon(j)$ was calculated using an Euclidean norm. The correlation dimension was estimated as the slope of $\ln (j)$ versus $\ln [\langle\varepsilon(j)\rangle]$ within a certain scaling region, in which this slope is well defined and may be calculated using a least-squares fit. The angle brackets indicate averaging over all reference vectors. All the results shown below were obtained using $N=10000, M=400$, and $K=10$ vectors. A large number of tests were carried out to find the proper values of the sampling time $\Delta T$ and of the delay time $\tau$. Their values depended on the case and are listed in captions of relevant figures. A criterion of choosing them was the best (linear in a wide range) scaling region and a saturation with increasing embedding dimension $m$ [14]. Figure 4 shows the examples of the examination of this saturation for the
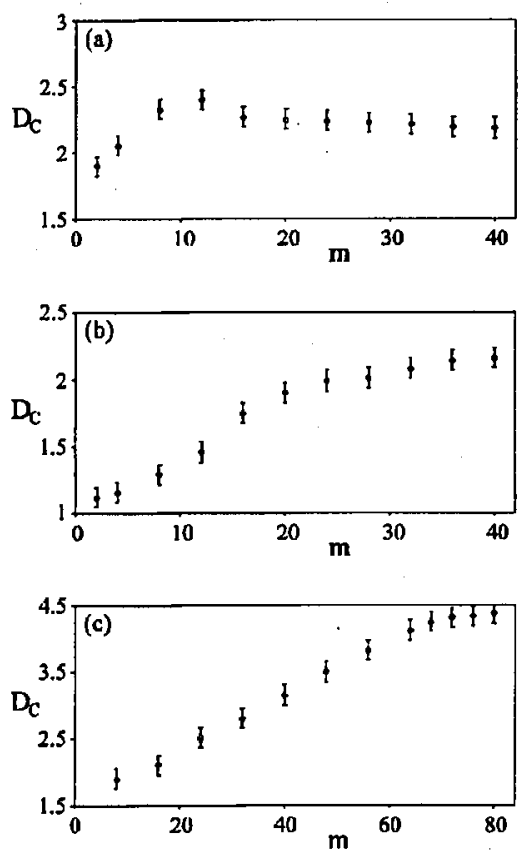

Fig. 4. Correlation dimension $D_{\mathrm{C}}$ as a function of embedding dimension $m$ obtained from the time series taken at the center of the systems for (a) $\mu_{x}\left(t_{i}\right)$ variable of Eqs. (1) with $H_{0}=40 \mathrm{Oe}, \Delta T=0.04 \mathrm{~ns}, \tau=4 \Delta T$, (b) $|\psi|\left(t_{i}\right)$ variable of Eq. (3) with periodic boundary conditions and $\alpha=0.149, \Delta T=0.15, \tau=4 \Delta T$, (c) $|\psi|\left(t_{i}\right)$ variable of Eq. (4) with periodic boundary conditions and $c_{2}=1.25, \Delta T=3.6, \tau=4 \Delta T$. Error bars are shown. 
three systems. The error of the values of the correlation dimension is estimated to be about $2.5 \%$ (5\% for Eq. (4)) in the chaotic states and it is probably smaller in the periodic ones. To estimate this error the algorithm was run some times for the same chosen values of the parameters and the half of the difference between the highest and the smallest values of the correlation dimension obtained was taken as the error.

The correlation dimension was found to be equal to 1 for all checked 1-periodic states of Eqs. (1) and Eq. (3), and equal to 2 for 2-periodic states of Eqs. (1). Figure 5 depicts $\ln (j)$ versus $\ln [\langle\varepsilon(j)\rangle]$ for 1-periodic and 2-periodic states shown in Figs. 1a, 4. The scaling regions are well defined in these cases and one can easily find that the slopes have proper values, 1 and 2 , respectively. These results con-
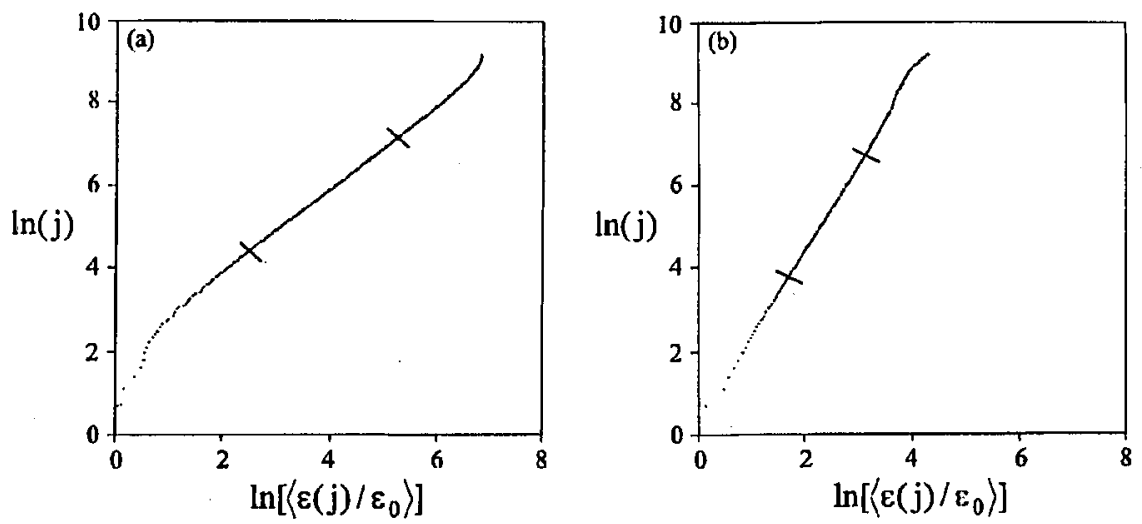

Fig. 5. Dependence of $\ln (j)$ on $\ln \left[\left\langle\varepsilon(j) / \varepsilon_{0}\right\rangle\right]$ (where $\varepsilon_{0}$ is the smallest from the $\varepsilon(j)$ values) obtained for $H_{0}=32.7$ Oe from a time series $\mu_{x}\left(t_{i}\right)$ at grid points number (a) 0, (b) 58. Bars indicate the scaling regions. The values of the algorithm parameters are: $m=30, \Delta T=0.032 \mathrm{~ns}, \tau=4 \Delta T$.

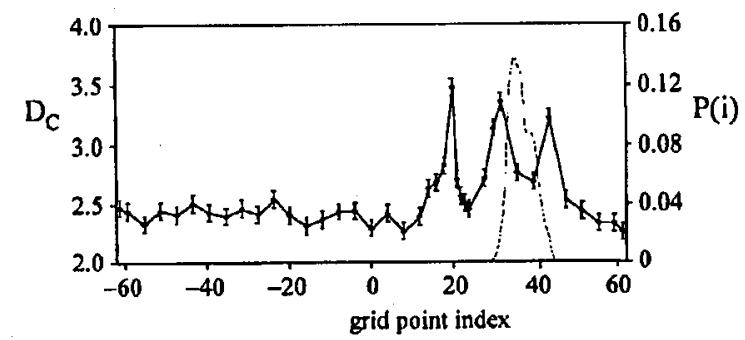

Fig. 6. Correlation dimension $D_{\mathrm{C}}$ for Eqs. (1) with $H_{0}=40$ Oe as a function of position along the $x$ axis. The correlation dimension was obtained from time series $\mu_{x}\left(t_{i}\right)$ with the following values of the algorithm parameters: $m=40, \Delta T=0.04 \mathrm{~ns}, \tau=4 \Delta T$. Error bars are shown. The dashed curve denotes the probability $P(i)$ that the centre of the wall is at grid point number $i$. 
firm what was previously obtained using Fourier transform and suggest that the correlation dimension calculated, on the basis of the time-delay technique, from a time series taken at one grid point is a good measure of the local properties of the attractor. Therefore, it can be a tool to examine the spatial inhomogeneity of a system, especially in the chaotic state, when the Fourier transform does not seem to be useful. The results of using the local correlation dimension as such a tool
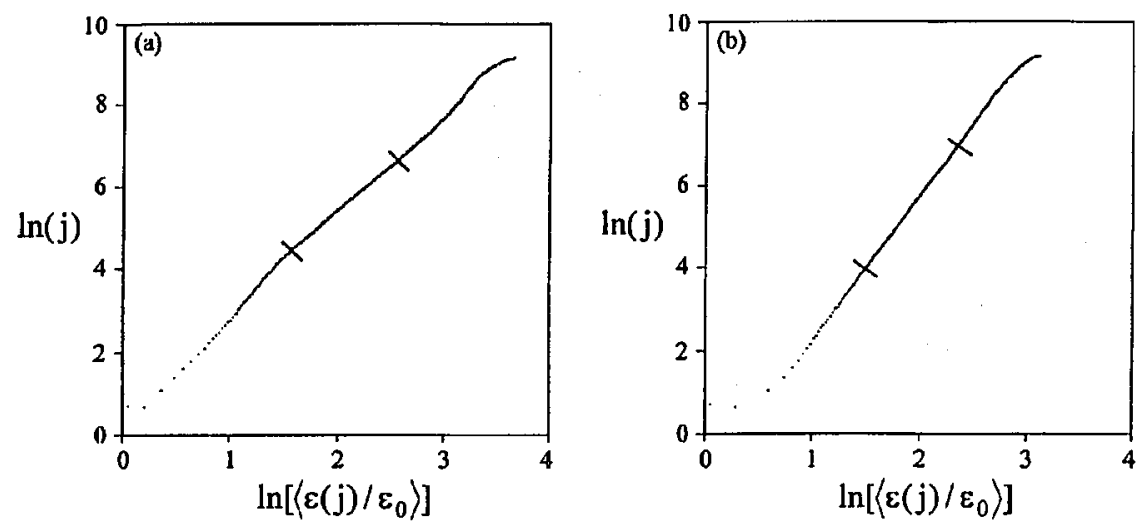

Fig. 7. Dependence of $\ln (j)$ on $\ln \left[\left\langle\varepsilon(j) / \varepsilon_{0}\right\rangle\right]$ (where $\varepsilon_{0}$ is the smallest from the $\varepsilon(j)$ values) obtained for $H_{0}=40$ Oe from a time series $\mu_{x}\left(t_{i}\right)$ at grid points number (a) $0\left(D_{\mathrm{C}} \approx 2.3\right)$, (b) $32\left(D_{\mathrm{C}} \approx 3.3\right)$. Bars indicate the scaling regions. The values of the algorithm parameters are the same as in Fig. 6.


Fig. 8. Correlation dimension $D_{\mathrm{C}}$ for Eq. (3) with $\alpha=0.149$ as a function of position along the $x$ axis. The lower curve is related to the periodic boundary conditions and the upper one - to the pinned boundary conditions. The correlation dimension was obtained from time series $|\psi|\left(t_{i}\right)$ with the following values of the algorithm parameters: $m=40, \Delta T=0.15, \tau=4 \Delta T$. Error bars are shown.

Fig. 9. Correlation dimension $D_{\mathrm{C}}$ as a function of position along the $x$ axis for Eq. (4) with periodic boundary conditions and for $c_{2}=1.25$ (squares), $c_{2}=3.0$ (triangles). The correlation dimension was obtained from time series $|\psi|\left(t_{i}\right)$ with the following values of the algorithm parameters: $m=80, \Delta T=3.6$ for $c_{2}=1.25$ and $\Delta T=0.18$ for $c_{2}=3.0, \tau=4 \Delta T$. The vertical size of the markers is related to the error. 
are shown in Figs. 6, 8, 9, which depict the correlation dimension as a function of position along the $x$ axis. Figure 6. depicts this function for Eqs. (1) and for $H_{0}=40$ Oe. For this value of the amplitude of the driving field the wall moves back and forth within a small range of the space, the probability $P(i)$ that the center of the wall is at grid point number $i$ is shown in Fig. 6 by a dashed curve. From Fig. 6, a clear interrelation between the position of the wall and the value of the local correlation dimension is seen, beyond the range of the wall residence the value of the correlation dimension changes only very slightly from one position to another, while within the wall and in its neighborhood significantly larger differences between the values of the correlation dimension at different positions occur. Figure 7 shows $\ln (j)$ versus $\ln [\langle\varepsilon(j)\rangle]$ for two points with considerably different values of the correlation dimension. In Fig. 8 the correlation dimension is seen as a function of position along the $x$ axis for Eq. (3) $(\alpha=0.149)$ with periodic and pinning boundary conditions. In these cases the interrelation between the position of the solitary wave (which is placed at the center of the system) and the values of the local correlation dimension correlation is also seen. Moreover, one can see the influence of the boundaries on the value of the correlation dimension. Figure 9 depicts the correlation dimension as a function of position along the $x$ axis for Eq. (4), for two values of the nonlinearity parameter $c_{2}=1.25$ (phase turbulence regime) and $c_{2}=3.0$ (amplitude turbulence regime). It is seen that the attractors are spatially homogeneous. Besides, the value of the correlation dimension is the same (within an error) $D_{\mathrm{C}} \approx 4.2$ for both, the phase and amplitude turbulence regimes. The same values of the correlation dimension were found for all checked values of $c_{2}$. Figure 9 was obtained for periodic boundary conditions, but qualitatively the same results were obtained for pinning boundary conditions, only the value of the correlation dimension was larger, $D_{\mathrm{C}} \approx 4.5$.

\section{Conclusion}

For the three systems the spatial inhomogeneity was examined on the basis of the Takens delay-time reconstruction method applied for different positions in space. The systems described by the Landau-Lifshitz equation and the driven damped nonlinear Schrödinger equation are found to be spatially non-uniform, both in non-chaotic and chaotic states. It was found both by the Fourier transform for the periodic and quasi-periodic states as well as by the calculation, using time-delay method, of the local correlation dimension for the chaotic states. In the case of these two systems the spatial inhomogeneity is mostly related to the existence of spatially coherent structures in the form of solitary waves. On the other hand, the third system described by the complex Ginzburg-Landau equation, which is the system without solitary waves, was found to be spatially uniform.

In conclusion, the reconstruction of the attractor" by the time-delay method may give qualitatively different results for time series obtained at different space positions of the inhomogeneous spatially extended systems. "Reconstructed" attractors do not reflect the global dynamics of such systems, but they allow one to describe their local properties. In particular, the values of the local correlation dimension may characterize the spatial inhomogeneity of the chaotic states. 


\section{Acknowledgment}

One of us (A.S.) was supported by the Committee for Scientific Research under grant No. 2 PO3B 04208.

\section{References}

[1] F. Takens, Dynamical Systems and Turbulence, in series Springer Lecture Notes in Mathematics, Eds. D.A. Rand, L.-S. Young, Vol. 898, Springer-Verlag, Berlin 1981, p. 366.

[2] P. Grassberger, I. Procaccia, Physica D 9, 189 (1983).

[3] J.J. Żebrowski, Phys. Rev. E 47, 2308 (1993).

[4] A. Zubrzycki, J. Magn. Magn. Mater. 151, 290 (1995).

[5] N. Bekki, K. Nozaki, in: Dynamical Problems in Soliton Systems, Springer Series in Synergetics, Ed. S. Takeno, Vol. 30, Springer Verlag, Berlin 1985, p. 268.

[6] W. Schöpf, L. Kramer, Phys. Rev. Lett. 66, 2316 (1991).

[7] T. Bohr, A.W. Pedersen, M.II. Jensen, Phys. Rev. A 42, 3626 (1990).

[8] B.I. Shraiman, A. Pumir, W. Van Saarloos, P.C. Hohenberg, H. Chate, M. Holen, Physica D 57, 241 (1992).

[9] D.A. Egolf, H.S. Greenside, Nalure 369, 129 (1994).

[10] B. Fornberg, G.B. Whitham, Philos. Trans. R. Soc. 289, 373 (1978).

[11] N.L. Schryer, L.R. Walker, J. Appl. Phys. 45, 5406 (1974).

[12] A. Zubrzycki, Acta Phys. Pol. A 88, 1067 (1995).

[13] A. Zubrzycki, Acta Phys. Pol. A 87, 925 (1995).

[14] J.W. Havstad, C.L. Ehlers, Phys. Rev. A 39, 845 (1989).

[15] J. Theiler, Phys. Rev. A 34, 2427 (1986). 